Tôhoku Math. Journ. 22(1970), 109-116.

\title{
SOME REMARKS ON WIENER'S QUASI-TAUBERIAN THEOREMS AND THE SUMMABILITY OF FOURIER SERIES
}

\author{
Kôzô YABUTA
}

(Received September 11, 1969)

In [5] N. Wiener gave the so-called quasi-tauberian theorems and their applications to the problem on the summability of Fourier series and integrals by Cesàro sum of some order, which was discussed completely by L. Bosanquet [1] and R. Paley [3]. But we have found that Wiener's discussion on kernels vanishing for positive arguments is not correct, and that his Theorem $22^{\prime}$ is not stated correctly. In his Theorem 22' the phrase $-\varepsilon<\operatorname{Re} u<\lambda+\varepsilon$ must be replaced by $-\lambda-\varepsilon<\operatorname{Re} u<\varepsilon$. The result of Bosanquet-Paley reads as follows;

THEOREM. Let $f(x)$ be an integrable function of period $2 \pi$ and let

$$
\phi(x)=\frac{1}{2}(f(x+y)+f(y-x)-2 s) .
$$

Then if we write $B_{m}$ for the proposition

$$
\lim _{\varepsilon \rightarrow 0} \frac{1}{\varepsilon} \int_{0}^{\varepsilon}\left(1-\frac{x}{\varepsilon}\right)^{m-1} \phi(x) d x=0
$$

and $C_{m}$ for the proposition

$$
\lim _{x \rightarrow 0} \frac{1}{\varepsilon} \int_{0}^{1} \dot{\phi}(x) d x \int_{0}^{1}(1-z)^{m} \cos \frac{x z}{\varepsilon} d z=0,
$$

$B_{m}$ implies $C_{m+\varepsilon}$ for $m \geqq 0$, while $C_{m}$ implies $B_{m+1+\varepsilon}$ for $m \geqq 0$ and any $\varepsilon>0$.

If we apply Theorem 22' of Wiener [5], we can obtain only that $B_{m}$ implies $C_{m+\varepsilon}$ when $m \geqq 1$ and that $C_{m}$ implies $B_{m+2+\varepsilon}$ when $m \geqq 0$. But applying Levinson's variant of Theorem 22' of Wiener [2] and another quasi-tauberian theorem for some unbounded kernel, we can reprove the Bosanquet-Paley's result completely by Wiener's method. By his method we have also reproved F.T.Wang's results on the summability of Fourier series by Riesz's logarithmic means [4].

To prove Theorem we need two quasi-tauberian theorems. 
LEMMA 1. (Wiener-Levinson) Let

$$
\lim _{\varepsilon \rightarrow 0} \frac{1}{\varepsilon} \int_{0}^{1} N_{1}\left(\begin{array}{l}
x \\
\varepsilon
\end{array}\right) \phi(x) d x=0
$$

where

$$
\int_{0}^{1}|\phi(x)| d x<\infty
$$

and

$$
\left|N_{1}(x)\right|<A<\infty \text {. }
$$

If $R(x)$ is a function such that

$$
\int_{0}^{\infty}|R(x)| d x<\infty
$$

and

$$
\int_{0}^{\infty} \frac{|R(x)|}{x} d x<\infty
$$

and if

$$
N_{2}(x)=\int_{0}^{\infty} R(y) N_{1}\left(\frac{x}{y}\right) \frac{d y}{y},
$$

then we have

$$
\lim _{\varepsilon \rightarrow 0} \frac{1}{\varepsilon} \int_{0}^{1} N_{2}\left(\frac{x}{\varepsilon}\right) \phi(x) d x=0 .
$$

LEMMA 2. Lemma 1 remains valid if (2) and (3) are replaced by

$$
\int_{0}^{\infty}\left|N_{1}(x)\right| d x<\infty
$$

(8) there exist $K>0$ and $A>0$ such that

$$
\left|N_{1}(x)\right|<A \text { for } 0<x<K \text {, }
$$

$$
|R(x)|<B<\infty
$$


and

$$
R(x)=O\left(1 / x^{1+\alpha}\right) \text { as } x \rightarrow \infty \text { for some } \alpha>0
$$

i.e., there exists $C>0$ such that $|R(x)|<C / x^{1+\alpha}$.

PROOF OF LEMMA 2. By simple calculation, the following repeated integral is absolutely convergent, so we can interchange the order of integration, giving

$$
\begin{aligned}
& \frac{1}{\varepsilon} \int_{0}^{\infty} R\left(\frac{y}{\varepsilon}\right) \frac{d y}{y} \int_{0}^{1} N_{1}\left(\frac{x}{y}\right) \phi(x) d x \\
& \quad=\frac{1}{\varepsilon} \int_{0}^{1} \phi(x) d x \int_{0}^{\infty} R\left(\frac{y}{\varepsilon}\right) N_{1}\left(\frac{x}{y}\right) \frac{d y}{y}=\frac{1}{\varepsilon} \int_{0}^{1} \phi(x) N_{2}\left(\frac{x}{\varepsilon}\right) d x
\end{aligned}
$$

Since

$$
\left|\int_{\delta}^{\infty} R\left(\frac{y}{\varepsilon}\right) N_{1}\left(\frac{x}{y}\right) \frac{d y}{y}\right|<C \varepsilon^{1+\alpha} / \delta^{\alpha} x \int_{0}^{\infty}\left|N_{1}(t)\right| d t
$$

for every $\delta>0$, by (7), (8), (9), (10), it follows that

$$
\begin{aligned}
&\left|\frac{1}{\varepsilon} \int_{0}^{\infty} R\left(\frac{y}{\varepsilon}\right) \frac{d y}{y} \int_{0}^{1} N_{1}\left(\frac{x}{y}\right) \phi(x) d x\right| \\
& \leqq \frac{1}{\varepsilon} \int_{0}^{\delta} R\left(\frac{y}{\varepsilon}\right) d y\left\{\frac{1}{y} \int_{0}^{1} N_{1}\left(\frac{x}{y}\right) \phi(x) d x\right\} \mid \\
&+\left|\frac{1}{\varepsilon} \int_{K \delta}^{1} \phi(x) d x \int_{\delta}^{\infty} R\left(\frac{y}{\varepsilon}\right) N_{1}\left(\frac{x}{y}\right) \frac{d y}{y}\right| \\
&+\left|\frac{1}{\varepsilon} \int_{0}^{K \delta} \phi(x) d x \int_{\delta}^{\infty} R\left(\frac{y}{\varepsilon}\right) N_{1}\left(\frac{x}{y}\right) \frac{d y}{y}\right| \\
& \leqq\left|\frac{1}{\varepsilon} \int_{0}^{\delta} R\left(\frac{y}{\varepsilon}\right) d y\left\{\frac{1}{y} \int_{0}^{1} N_{1}\left(\frac{x}{y}\right) \phi(x) d x\right\}\right| \\
&+\frac{C \varepsilon^{\alpha} A}{\delta^{1+\alpha}} \int_{0}^{K \delta}|\phi(x)| d x+\frac{C \varepsilon^{\alpha}}{\delta^{\alpha}} \int_{K \delta}^{1} \frac{|\phi(x)|}{x} d x \int_{0}^{\infty}\left|N_{1}(x)\right| d x .
\end{aligned}
$$

Since for sufficiently small $\delta$ the first term on the right is arbitrarily small independently of $\varepsilon$, and any fixed $\delta$, the second term and the third are arbitrarily small, we have the conclusion considering (11). 
Now we shall first prove that $B_{m}$ implies $C_{m+\varepsilon}$ for $m \geqq 0$. It is clearly sufficient to prove for $m>0$, because $B_{n}$ implies $B_{n+\beta}$ for every $\beta>0$ and $n \geqq 0$. Let $n>m>0$ and

$$
\begin{aligned}
& N_{1}(x)=\frac{(1-x)^{m-1}}{\Gamma(m)} \quad 0<x<1 \quad(m>0) \\
& =0 \quad 1 \leqq x
\end{aligned}
$$

and

$$
N_{2}(x)=\frac{2}{\pi \Gamma(n+1)} \int_{0}^{1}(1-z)^{n} \cos z x d z \quad(n \geqq 0) .
$$

Their Mellin transforms are given by

$$
\begin{gathered}
n_{1}(w)=\int_{0}^{\infty} x^{-w} N_{1}(x) d x=\frac{\Gamma(1-w)}{\Gamma(m+1-w)} \\
n_{2}(w)=\int_{0}^{\infty} x^{-w} N_{2}(x) d x=\frac{1}{\Gamma(n+1+w) \cos \pi / 2 w} .
\end{gathered}
$$

Set $r(w)=\frac{n_{2}(w)}{n_{1}(w)}$. Then $r(w)$ is holomorphic in the half plane $\operatorname{Re} w<m+1$. And by Stirling's formula

$$
|r(w)| \sim \frac{2}{\sqrt{2 \pi}}|\operatorname{Im} w|^{-\operatorname{Re} w+m-n-1 / 2}
$$

as $\operatorname{Im} w \rightarrow \pm \infty$, uniformly in $-n-1<\operatorname{Re} w<m+1$. Hence $r(w)$ is uniformly $L^{2}$ in $-\frac{n-m}{2} \leqq \operatorname{Re} w \leqq 1+\frac{m}{2}$ and via Paley-Wiener theorem we have

$$
\int_{0}^{\infty}|R(x)| d x<\infty \text { and } \int_{0}^{\infty} \frac{|R(x)|}{x} d x<\infty
$$

where $R(x)=1$. i.m $\frac{1}{2 \pi i} \int_{-i A}^{i A} r(w) x^{w-1} d w$. This function $R(x)$ satisfies the integral equation (5). Thus, since $N_{1}(x)$ is bounded for $m \geqq 1$, Lemma 1 shows that $B_{m}$ implies $C_{m+\varepsilon}$ in this case. For the case $1>m>0$ we apply Lemma 2 . We see easily that $N_{1}(x)$ satisfies (7), (8) and that $R(x)$ satisfies (4). Hence we have only to show that $R(x)$ is bounded and $O\left(\frac{1}{x^{1+\alpha}}\right)$ for some $\alpha>0$. The 
boundedness follows immediately from the fact $r(1+i v) \in L^{1}(-\infty,+\infty)$, easily seen by (16). To see the order of growth, we need the following lemma which follows from Stirling's formula.

LEMMA 3. Let $a, b, c$ be three numbers such that $a \leqq c$ and $-a \leqq b$. Then

$$
\frac{\Gamma(b-w)}{\Gamma(w+a)}-(-1)^{a-c} \frac{\Gamma(b-a+c-w)}{\Gamma(w+c)}=\frac{\Gamma(b-w)}{\Gamma(w+a)} O(1 / w)
$$

and

$$
\frac{\Gamma(w+a)}{\Gamma(b-w)}-(-1)^{a-c} \frac{\Gamma(w+c)}{\Gamma(b-a+c-w)}=\frac{\Gamma(w+a)}{\Gamma(b-w)} O(1 / w)
$$

as $\operatorname{Im} w \rightarrow \pm \infty$, uniformly in $-a \leqq \operatorname{Re} w \leqq b$.

Set

$$
r_{1}(w)=(-1)^{m} \frac{1}{\Gamma(n-m+1+w) \cos \pi / 2 w}=\int_{0}^{\infty} x^{-w} R_{1}(x) d x
$$

where

$$
R_{1}(x)=\frac{(-1)^{m} 2}{\pi \Gamma(n-m+1)} \int_{0}^{1}(1-z)^{n-m} \cos z x d z .
$$

Applying Lemma 3 to $r(w)$ and $r_{1}(w)$, we have

$$
r(w)-r_{1}(w)=O\left(|\operatorname{Im} w|^{-\mathrm{R} \theta+m-n-3 / 2}\right) .
$$

Hence $r(w)-r_{1}(w)$ is uniformly $L^{2}$ and $L^{1}$ in $-\frac{1}{2} \leqq \operatorname{Re} w \leqq \frac{1}{2}$. Since $r(w)-r_{1}(w)$ is holomorphic in $-1<\operatorname{Re} w<1$, there exists $C>0$ such that

$$
\left|R(x)-R_{1}(x)\right|<C x^{-3 / 2} .
$$

By simple calculation we have

$$
R_{1}(x)=O\left(x^{-1-\beta_{1}}\right),
$$

where $\beta_{1}=\inf (1, n-m)$, 
which, combined with (19), shows that

$$
R(x)=O\left(1 / x^{1+\beta_{2}}\right),
$$

where $\beta_{2}=\inf \left(\beta_{1}, \frac{1}{2}\right)$.

We have thus proved the first half of Theorem.

Next we shall show that $C_{m}$ implies $B_{m+1+\varepsilon}$ for $m \geqq 0$. In this case, if we have (11) interchanging the rolls of $N_{1}(x)$ and $N_{2}(x)$, the proof goes in the same way as in Lemma 1 (Levinson [2], p. 140), because $N_{2}(x)$ is bounded for $n \geqq 0$. Let $m-1>n \geqq 0$ and

$$
r(w)=\frac{n_{1}(w)}{n_{2}(w)}=\frac{\Gamma(1-w) \Gamma(n+1+w) \cos \pi / 2 w}{\Gamma(m+1-w)}
$$

and

$$
\begin{aligned}
r_{1}(w) & =\frac{(-1)^{n+1} \Gamma(1-w) \Gamma(1+w) \cos \pi / 2 w}{\Gamma(m-n+1-w)} \\
& =\int_{0}^{\infty} x^{-w} R_{1}(x) d x
\end{aligned}
$$

where

$$
\begin{aligned}
R_{1}(x)= & \frac{(-1)^{n+1}}{\Gamma(m-n)}\left\{1 / x \int_{0}^{1}(1-z)^{m-n-1} \cos \frac{z}{x} d z\right. \\
& \left.-\frac{m-n-1}{x} \int_{0}^{1} z(1-z)^{m-n-2} \cos \frac{z}{x} d z\right\} \\
= & \frac{(-1)^{n+1}}{\Gamma(m-n)}\left\{R_{2}(x)-R_{3}(x)\right\} .
\end{aligned}
$$

We shall show that we have (11) if we replace $R(x)$ by $R(x)-R_{1}(x)$ or $R_{1}(x)$ and thus (11) follows for $R(x)$. By Lemma 3, we have

$$
r(w)-r_{1}(w)=O\left(|\operatorname{Im} w|^{R e w+n-m-1 / 2}\right) .
$$

Since $r(w)-r_{1}(w)$ is holomorphic in $-1<\operatorname{Re} w<2$, via Paley-Wiener theorem we have

$$
R(x)-R_{1}(x), \quad\left(R(x)-R_{1}(x)\right) / x \in L^{1}(0, \infty) .
$$


In the same way we have

$$
R(x), R_{1}(x) \in L^{1}(0, \infty)
$$

Since $N_{2}(x)$ is bounded, we have (11) for $R(x)-R_{1}(x)$.

For $R_{2}(x)$ we have by simple calculation

$$
\begin{aligned}
R_{2}(x) & =O\left(x^{-1}\right) \quad(x \rightarrow \infty) \\
& =O\left(x^{\beta}\right) \quad(x \rightarrow 0),
\end{aligned}
$$

where $\beta=\inf (1, m-n-1)$. Thus $R_{2}(x) / x \in L^{1}(0, \infty)$, which shows that (11) remains valid for $R_{2}(x)$.

Now consider $R_{3}(x)$. Let $\delta$ be an arbitrarily fixed positive number and $k=m-n-1$. Let

$$
\begin{aligned}
I_{\delta}(x) & =\int_{\delta}^{\infty} R_{3}\left(\frac{y}{\varepsilon}\right) N_{2}\left(\frac{x}{y}\right) \frac{d y}{y} \\
& =\int_{\delta}^{\infty} \frac{d y}{y}\left(\int_{0}^{1} \frac{\varepsilon k z}{y}(1-z)^{k-1} \cos \frac{\varepsilon z}{y} d z\right)\left(\int_{0}^{1}(1-t)^{n} \cos \frac{x t}{y} d t\right) \\
& =\varepsilon k \int_{0}^{1} \int_{0}^{1} z(1-z)^{k-1}(1-t)^{n} d z d t \int_{\delta}^{\infty} \frac{\cos \varepsilon z / y \cos x t / y}{y^{2}} d y .
\end{aligned}
$$

The last term is equal to $\int_{0}^{1 / \delta} \cos \varepsilon_{z} y \cos x t y d y=\frac{1}{2} \int_{0}^{1 / \delta}\left\{\cos \left(\varepsilon_{z}+x t\right) y+\cos \left(\varepsilon_{z}\right.\right.$ $-x t) y\} d y$. Clearly it is less than or equal to 1 unless $\varepsilon z= \pm x t$. Hence $I_{\delta}(x)$ is finite with respect to $0<\delta<\infty$ and $0 \leqq x \leqq 1$. Hence applying Lebesgue's dominated convergence theorem and Fubini's theorem, we have

$$
\begin{aligned}
& \int_{0}^{\infty} R_{3}\left(\frac{y}{\varepsilon}\right) \frac{d y}{y} \int_{0}^{1} N_{2}\left(\frac{x}{y}\right) \phi(x) d x \\
& =\lim _{\delta \rightarrow 0} \int_{\delta}^{\infty} R_{3}\left(\frac{y}{\varepsilon}\right) \frac{d y}{y} \int_{0}^{1} N_{2}\left(\frac{x}{y}\right) \phi(x) d x \\
& =\lim _{\delta \rightarrow 0} \int_{0}^{1} \phi(x) d x \int_{\delta}^{\infty} R_{3}\left(\frac{y}{\varepsilon}\right) N_{2}\left(\frac{x}{y}\right) \frac{d y}{y} \\
& =\int_{0}^{1} \phi(x) d x \int_{0}^{\infty} R_{3}\left(\frac{y}{\varepsilon}\right) N_{2}\left(\frac{x}{y}\right) \frac{d y}{y},
\end{aligned}
$$


which shows that (11) holds for $R_{3}(x)$. Thus we have shown that (11) holds for $R(x)$, which completes the proof.

\section{REFERENCES}

[1] L.S. Bosanquet, On the summability of Fourier series, Proc. London Math. Soc., (2)31 (1930), 144-164.

[2] N. Levinson, On the Poisson summability of Fourier series, Duke Math. J., 2(1936), 138-146.

[3] R.E.A.C.PAley, On the Cesàro summability of Fourier series and allied series, Proc. Cambridge Philos. Soc., 26(1930), 173-203.

[4] F.T. WANG, On the summability of Fourier series by Riesz's logarithmic means, II, Tôhoku Math. J., 40(1935), 274-292.

[5] N. WIENER, Tauberian theorems, Ann. of Math., 32(1931), 1-100.

MATHEMATICAL INSTITUTE

TÔHOKU UNIVERSITY

SENDAI, JAPAN 